MSC 42B35, 26A33

\title{
CHARACTERIZATION OF BMO VIA BALL BANACH FUNCTION SPACES
}

\author{
Mitsuo Izuki ${ }^{1}$, Yoshihiro Sawano ${ }^{2}$ \\ 1 Okayama University, \\ 3-1-1 Tsushima-naka, Okayama, 700-8530 Japan \\ 2 Tokyo Metropolitan University, \\ 1-1 Minami-Ohsawa, Tokyo, Hachioji, 192-0397 Japan
}

The aim of this paper is to characterize the BMO norm via ball Banach function spaces based on the Rubio de Francia algorithm. The method in this paper can be applicable to the Campanato spaces. Refs 28 .

Keywords: BMO norm, ball Banach function spaces, Rubio de Francia algorithm, Campanato spaces.

1. Introduction. The BMO space is known as the dual space of the Hardy space $H^{1}\left(\mathbb{R}^{n}\right)$ and plays an important role in real analysis due to many important characterizations. The space $\operatorname{BMO}\left(\mathbb{R}^{n}\right)$ consists of all locally integrable functions $b$ satisfying that the semi-norm

$$
\|b\|_{\mathrm{BMO}}:=\sup _{Q: \text { cube }} \frac{1}{|Q|} \int_{Q}\left|b(x)-b_{Q}\right| d x
$$

is finite, where for each cube $Q \subset \mathbb{R}^{n},|Q|$ is the Lebesgue measure, and $b_{Q}$ is the mean value of the function $b$ on $Q$, namely

$$
b_{Q}:=\frac{1}{|Q|} \int_{Q} b(y) d y .
$$

The semi-norm $\|b\|_{\mathrm{BMO}}$ is called the $\mathrm{BMO}$ norm. If $b \in \mathrm{BMO}\left(\mathbb{R}^{n}\right)$, then there exist positive constants $C_{1}$ and $C_{2}$ such that for all cubes $Q$ and $\lambda>0$,

$$
\left|\left\{x \in Q:\left|b(x)-b_{Q}\right|>\lambda\right\}\right| \leqslant C_{1}|Q| \exp \left(-\frac{C_{2} \lambda}{\|b\|_{\mathrm{BMO}}}\right) .
$$

The inequality (1.1) is proved by John and Nirenberg [1] and implies that for any constant $1 \leqslant p<\infty$ there exists a constant $C \geqslant 1$ such that

$$
\|b\|_{\mathrm{BMO}_{L^{1}}} \leqslant\|b\|_{\mathrm{BMO}_{L^{p}}} \leqslant C\|b\|_{\mathrm{BMO}_{L^{1}}},
$$

where $\chi_{Q}$ is the characteristic function for $Q$ and

$$
\|b\|_{\mathrm{BMO}_{L^{p}}}:=\sup _{Q: \text { cube }} \frac{1}{\left\|\chi_{Q}\right\|_{L^{p}\left(\mathbb{R}^{n}\right)}}\left\|\left(b-b_{Q}\right) \chi_{Q}\right\|_{L^{p}\left(\mathbb{R}^{n}\right)} .
$$

Mitsuo Izuki was partially supported by Grand-in-Aid for Scientific Research (C), No. 15K04928, for Japan Society for the Promotion of Science.

Yoshihiro Sawano was partially supported by Grand-in-Aid for Scientific Research (C), No. 16K05209, for Japan Society for the Promotion of Science.

(C) Санкт-Петербургский государственный университет, 2017 
The aim of this paper is to replace $L^{p}\left(\mathbb{R}^{n}\right)$ by general function spaces having similar properties.

We work on ball Banach function spaces, whose definition we present now.

Definition 1.1. [2, Definition 2] Let $\mathcal{M}$ be the set of all complex-valued measurable functions defined on $\mathbb{R}^{n}$. A mapping $\rho: \mathcal{M} \rightarrow[0, \infty]$ is called a ball Banach function norm if, for all $f, g, f_{k},(k=1,2,3, \ldots)$, in $\mathcal{M}$, for all constants $a \geqslant 0$ and for all cubes $Q$ in $\mathbb{R}^{n}$, the following properties hold:

(P1) $\rho(f)=0$ if and only if $f=0$ a. e.; $\rho(a f)=a \rho(f) ; \rho(f+g) \leqslant \rho(f)+\rho(g)$;

(P2) If $0 \leqslant g \leqslant f$ a. e., then $\rho(g) \leqslant \rho(f)$;

(P3) If $0 \leqslant f_{k} \uparrow f$ a. e. then $\rho\left(f_{k}\right) \uparrow \rho(f)$;

(P4)' If $|Q|<\infty$, then $\rho\left(\chi_{Q}\right)<\infty$;

(P5)' If $f \geqslant 0$ a.e. and $|Q|<\infty$, then $\int_{Q} f(x) d x \leqslant C_{Q} \rho(f)$ for some constant $C_{Q}$, $0<C_{Q}<\infty$, depending on $Q$ and $\rho$ but independent of $f$.

The definition remains unchanged if we replace "cube" by "ball" in the above. So this definition deserves this name.

Accrodingly, the space generated by such $\rho$ is called the ball Banach function space.

Suppose that $X$ is a ball Banach function space equipped with a norm $\|\cdot\|_{X}$. The associate space $X^{\prime}$ is defined by

$$
X^{\prime}:=\left\{f \in \mathcal{M}:\|f\|_{X^{\prime}}<\infty\right\},
$$

where

$$
\|f\|_{X^{\prime}}:=\sup \left\{\left|\int_{\mathbb{R}^{n}} f(x) g(x) d x\right|:\|g\|_{X} \leqslant 1\right\} .
$$

By using a similar technique in [3], we see that $X^{\prime}$ is a ball Banach function space as well. We also recall that the Hardy-Littlewood maximal operator $M$ is given by

$$
M f(x):=\sup _{Q: \text { cube }} \frac{\chi_{Q}(x)}{|Q|} \int_{Q}|f(y)| d y .
$$

In this paper we aim to provide a sufficient condition to characterize the BMO norm in terms of $X, X^{\prime}$ and $M$.

Theorem 1.1. Let $X$ be a ball Banach function space. If the Hardy-Littlewood maximal operator $M$ is bounded on the associate space $X^{\prime}$, then there exist positive constants $C_{1} \leqslant C_{2}$ such that for all $b \in \mathrm{BMO}\left(\mathbb{R}^{n}\right)$,

$$
C_{1}\|b\|_{\text {BMO }} \leqslant \sup _{Q: \text { cube }} \frac{1}{\left\|\chi_{Q}\right\|_{X}}\left\|\left(b-b_{Q}\right) \chi_{Q}\right\|_{X} \leqslant C_{2}\|b\|_{\text {BMO }}
$$

The quantity

$$
\|b\|_{\mathrm{BMO}_{X}}:=\sup _{Q: \text { cube }} \frac{1}{\left\|\chi_{Q}\right\|_{X}}\left\|\left(b-b_{Q}\right) \chi_{Q}\right\|_{X}
$$

is the $X$-based generalized BMO, which is one of our targets in this paper. Ho's proof [4] is based on the theory of Hardy spaces. We will give another proof of Theorem 1.1 using the Rubio de Francia algorithm. Our result is based on the following inequalities. 
Theorem 1.2. Let $X$ be a ball Banach function space such that the Hardy-Littlewood maximal operator $M$ is bounded on the associate space $X^{\prime}$. Then we have

$$
C^{-1} \frac{1}{|Q|} \int_{Q}|f(x)| d x \leqslant \frac{1}{\left\|\chi_{Q}\right\|_{X}}\left\|f \chi_{Q}\right\|_{X} \leqslant C\left(\frac{1}{|Q|} \int_{Q}|f(x)|^{p} d x\right)^{\frac{1}{p}}
$$

for some $1<p<\infty$.

Note that (1.4) is a consequence of Theorem 1.2 and (1.2).

Let $\theta \in(0,1)$ and $1 \leqslant p<\infty$. As an application of Theorem 1.2 , we can characterize the Campanato space $\mathcal{L}^{p, \theta}\left(\mathbb{R}^{n}\right)$ as well. Recall that the Campanato space $\mathcal{L}^{p, \theta}\left(\mathbb{R}^{n}\right)$ is the set of all $f \in L_{\text {loc }}^{p}\left(\mathbb{R}^{n}\right)$ for which the quantity

$$
\|f\|_{\mathcal{L}^{p, \theta}\left(\mathbb{R}^{n}\right)}:=\sup _{Q: \operatorname{cube}} \ell(Q)^{-\theta}\left(\frac{1}{|Q|} \int_{Q}\left|f(x)-f_{Q}\right|^{p} d x\right)^{\frac{1}{p}}
$$

is finite, where $\ell(Q)$ is the side length of $Q$. We know that there exists a constant $c_{\theta, p}$ such that

$$
\|f\|_{\mathcal{L}^{1, \theta}\left(\mathbb{R}^{n}\right)} \leqslant\|f\|_{\mathcal{L}^{p, \theta}\left(\mathbb{R}^{n}\right)} \leqslant c_{p}\|f\|_{\mathcal{L}^{1, \theta}\left(\mathbb{R}^{n}\right)} .
$$

This equivalence dates back to the works by Campanato and Meyer (see [5, Theorem, p. 183] and [6, Theorem, p.718]), where both authors showed that these norms are equivalent to the $\operatorname{Lip}^{\theta}$ norm. See [7, p. 72] for an account of these facts. See also [8, Theorem 3.1].

Let $X$ be a ball Banach function space. We consider the quantity:

$$
\|f\|_{\mathcal{L}^{X, \theta}\left(\mathbb{R}^{n}\right)}:=\sup _{Q: \text { cube }} \ell(Q)^{-\theta} \frac{\left\|\chi_{Q}\left(f-f_{Q}\right)\right\|_{X}}{\left\|\chi_{Q}\right\|_{X}} .
$$

Theorem 1.3. Let $1 \leqslant p<\infty, \theta \in(0,1)$ and $X$ be a ball Banach function space. If the Hardy-Littlewood maximal operator $M$ is bounded on the associate space $X^{\prime}$, then there exist positive constants $C_{1} \leqslant C_{2}$ such that for all $f \in \mathcal{L}^{1, \theta}\left(\mathbb{R}^{n}\right)$,

$$
C_{1}\|f\|_{\mathcal{L}^{X, \theta}\left(\mathbb{R}^{n}\right)} \leqslant\|f\|_{\mathcal{L}^{1, \theta}\left(\mathbb{R}^{n}\right)} \leqslant C_{2}\|f\|_{\mathcal{L}^{X, \theta}\left(\mathbb{R}^{n}\right)} .
$$

Note that (1.6) is a consequence of Theorem 1.2 and (1.5). Also, from the general pointwise estimate in Theorem 1.2, we learn that a passage to generalized Campanato spaces and to higher order Campanato spaces are also possible.

We work on ball Banach function spaces instead of Banach function spaces. We recall the definition of Banach function spaces to explain that Morrey spaces do not fall under the scope of Banach function spaces.

Definition 1.2. Let $X$ be a linear subspace of $\mathcal{M}$.

1. The space $X$ is said to be a Banach function space if there exists a functional $\|\cdot\|_{X}$ : $\mathcal{M} \rightarrow[0, \infty]$ satisfying the following properties. Let $f, g, f_{j} \in \mathcal{M}(j=1,2, \cdots)$, then

(a) $f \in X$ holds if and only if $\|f\|_{X}<\infty$;

(b) norm property: 
(i) positivity: $\|f\|_{X} \geqslant 0$;

(ii) strict positivity: $\|f\|_{X}=0$ holds if and only if $f(x)=0$ for almost every $x \in \mathbb{R}^{n}$

(iii) homogeneity: $\|\lambda f\|_{X}=|\lambda| \cdot\|f\|_{X}$ holds for all complex numbers $\lambda$;

(iv) triangle inequality: $\|f+g\|_{X} \leqslant\|f\|_{X}+\|g\|_{X}$;

(c) symmetry: $\|f\|_{X}=\||f|\|_{X}$;

(d) lattice property: if $0 \leqslant g(x) \leqslant f(x)$ for almost every $x \in \mathbb{R}^{n}$, then $\|g\|_{X} \leqslant\|f\|_{X}$;

(e) fatou property: if $0 \leqslant f_{j}(x) \leqslant f_{j+1}(x)$ for all $j$ and $f_{j}(x) \rightarrow f(x)$ as $j \rightarrow \infty$ for almost every $x \in \mathbb{R}^{n}$, then $\lim _{j \rightarrow \infty}\left\|f_{j}\right\|_{X}=\|f\|_{X}$;

(f) for every measurable set $F \subset \mathbb{R}^{n}$ such that $|F|<\infty,\left\|\chi_{F}\right\|_{X}$ is finite. Additionally there exists a constant $C_{F}>0$ depending only on $F$ such that for all $h \in X$,

$$
\int_{F}|h(x)| d x \leqslant C_{F}\|h\|_{X}
$$

Remark 1.1. In other literatures (for example [9]) the Banach function spaces and the associate space are called the Köthe space and the Köthe dual respectively.

The usual Lebesgue space $L^{p}\left(\mathbb{R}^{n}\right)$ with constant exponent $1 \leqslant p \leqslant \infty$ is an example of Banach function spaces. However, Morrey spaces are not Banach function spaces in general. When $1 \leqslant q<p<\infty$, then $\mathcal{M}_{q}^{p}\left(\mathbb{R}^{n}\right)$ is a ball Banach function space trivially but is not a Banach function space [10, Example 3.3]. In [10, Theorem 4.1] the second author and Tanaka showed that the associate space of the ball Banach space $\mathcal{M}_{q}^{p}\left(\mathbb{R}^{n}\right)$ is $\mathcal{H}_{q^{\prime}}^{p^{\prime}}\left(\mathbb{R}^{n}\right)$, where $\mathcal{H}_{q^{\prime}}^{p^{\prime}}\left(\mathbb{R}^{n}\right)$ is the block space defined by Zorko [11]. According to [12, Theorem 4.1], the Hardy-Littlewood maximal operator is bounded on $\mathcal{H}_{q^{\prime}}^{p^{\prime}}\left(\mathbb{R}^{n}\right)$ as long as $1<q \leqslant p<\infty$.

We organize the remaining part of this paper as follows: In Section 2, we review preliminary facts on ball Banach function spaces and on the Muckenhoupt weights. We prove Theorem 1.2 in Section 3. In Section 4, we consider some examples of $X$ together with related results.

2. Preliminaries. We describe some of fundamental facts of ball Banach function spaces, whose proof is similar to the one corresponding to Banach function spaces; see Bennett and Sharpley [3]. For further informations on the theory of Banach function spaces including the proof of Lemma 2.1 below we refer to the book [3].

Lemma 2.1. Let $X$ be a ball Banach function space. Then the following hold:

1) (The Lorentz-Luxemburg theorem) $\left(X^{\prime}\right)^{\prime}=X$ holds, in particular, the norms $\|\cdot\|_{\left(X^{\prime}\right)^{\prime}}$ and $\|\cdot\|_{X}$ are equivalent;

2) (The generalized Hölder inequality) If $f \in X$ and $g \in X^{\prime}$, then we have

$$
\int_{\mathbb{R}^{n}}|f(x) g(x)| d x \leqslant\|f\|_{X}\|g\|_{X^{\prime}} .
$$

Under a certain condition on the boundedness of the Hardy-Littlewood maximal operator $M$ on $\mathrm{X}$, the norm $\|\cdot\|_{X}$ enjoys properties similar to the Muckenhoupt weights. 
Lemma 2.2. Let $X$ be a ball Banach function space. Suppose that the HardyLittlewood maximal operator $M$ is weakly bounded on $X$, that is, there exists a positive constant $C$ such that

$$
\left\|\chi_{\{M f>\lambda\}}\right\|_{X} \leqslant C \lambda^{-1}\|f\|_{X}
$$

is true for all $f \in X$ and $\lambda>0$. Then we have

$$
\sup _{Q: \text { cube }} \frac{1}{|Q|}\left\|\chi_{Q}\right\|_{X}\left\|\chi_{Q}\right\|_{X^{\prime}}<\infty
$$

Proof. The proof is similar to the first author's papers [13, Lemmas 2.4 and 2.5] and [14, Lemmas G' and H]. For readers' convenience we give the self-contained proof. Take a cube $Q$ and a function $f \in L_{\text {loc }}^{1}\left(\mathbb{R}^{n}\right)$. Suppose that $|f|_{Q}>0$. Because $|f|_{Q} \chi_{Q}(x) \leqslant$ $M\left(f \chi_{Q}\right)(x)$ holds for almost every $x \in \mathbb{R}^{n}$, we obtain $M\left(f \chi_{Q}\right)(x)>\lambda$ for almost every $x \in Q$, where $\lambda:=|f|_{Q} / 2$. Hence by assumption (2.1) we get

$$
|f|_{Q}\left\|\chi_{Q}\right\|_{X} \leqslant|f|_{Q}\left\|\chi_{\left\{M\left(f \chi_{Q}\right)>\lambda\right\}}\right\|_{X} \leqslant|f|_{Q} \cdot C \lambda^{-1}\left\|f \chi_{Q}\right\|_{X}=2 C\left\|f \chi_{Q}\right\|_{X} .
$$

Therefore we have

$$
\begin{aligned}
\frac{1}{|Q|}\left\|\chi_{Q}\right\|_{X}\left\|\chi_{Q}\right\|_{X^{\prime}}= & \frac{1}{|Q|}\left\|\chi_{Q}\right\|_{X} \cdot \sup \left\{\int_{\mathbb{R}^{n}}|g(x)| \chi_{Q}(x) d x: g \in X,\|g\|_{X} \leqslant 1\right\}= \\
& =\sup \left\{|g|_{Q}\left\|\chi_{Q}\right\|_{X}: g \in X,\|g\|_{X} \leqslant 1\right\} \leqslant \\
& \leqslant \sup \left\{2 C\left\|g \chi_{Q}\right\|_{X}: g \in X,\|g\|_{X} \leqslant 1\right\} \leqslant 2 C .
\end{aligned}
$$

Remark 2.1. If $M$ is bounded on $X$, that is, there exists a positive constant $C$ such that

$$
\|M f\|_{X} \leqslant C\|f\|_{X}
$$

holds for all $f \in X$, then one can easily check that (2.1) holds. On the other hand, if $M$ is bounded on the associate space $X^{\prime}$, then Lemma 2.1 shows that (2.2) is true.

Next, we recall the notion of weights. Let $w$ be a locally integrable and positive function on $\mathbb{R}^{n}$. The function $w$ is said to be a Muckenhoupt $A_{1}$ weight if there exists a positive constant $C_{1}$ such that $M w(x) \leqslant C_{1} w(x)$ holds for almost every $x \in \mathbb{R}^{n}$. The set $A_{1}$ consists of all Muckenhoupt $A_{1}$ weights. For every $w \in A_{1}$, the finite value

$$
[w]_{A_{1}}:=\sup _{Q: \text { cube }}\left\{\frac{1}{|Q|} \int_{Q} w(x) d x \cdot\left\|w^{-1}\right\|_{L^{\infty}(Q)}\right\}
$$

is said to be a Muckenhoupt $A_{1}$ constant.

We remark that if $w \in A_{1}$, then

$$
\frac{1}{|Q|} \int_{Q} w(x) d x \leqslant[w]_{A_{1}} \inf _{x \in Q} w(x)
$$

for all cubes $Q$. We will use a classical result on the Muckenhoupt weights. 
Lemma 2.3. [15, Chapter 7; 16, Chapter 9] Let $w \in A_{1}$. We write $w(Q):=$ $\int_{Q} w(x) d x$ for a cube $Q$. Then the reverse Hölder inequality holds, that is, there exist positive constants $q>1$ and $C$ depending on $n$ and $[w]_{A_{1}}$ such that for all cubes $Q$,

$$
\left(\frac{1}{|Q|} \int_{Q} w(x)^{q} d x\right)^{1 / q} \leqslant C w_{Q}
$$

Actually, we know that the pair

$$
C=2, \quad q=1+\frac{1}{2^{n+3}[w]_{A_{\infty}}}
$$

does the job, where $[w]_{A_{\infty}}$ is the smallest number $B>0$ a for which

$$
w_{Q} \leqslant B \exp \left(\frac{1}{|Q|} \int_{Q} \log w(x) d x\right)
$$

for every cube $Q$. Note that $[w]_{A_{\infty}} \leqslant[w]_{A_{1}}$ for all weights $w$. This result can be found in [17, Theorem 2.3]; see [18, Theorem 4.2] for a generalization to spaces of homogeneous type.

\section{Proof of Theorem 1.2.}

Proof. We first prove the left-hand side inequality. Using Lemma 2.2 and Remark 2.1 , we get for all cubes $Q$,

$$
\frac{1}{|Q|} \int_{Q}|f(x)| d x \leqslant \frac{1}{|Q|}\left\|f \chi_{Q}\right\|_{X}\left\|\chi_{Q}\right\|_{X^{\prime}} \leqslant C \cdot \frac{1}{\left\|\chi_{Q}\right\|_{X}}\left\|f \chi_{Q}\right\|_{X},
$$

where $C>0$ is a constant independent of $f$ and $Q$. This shows the left-hand side inequality.

Next we prove the right-hand side inequality. Our idea is based on [19, Proof of Lemma 3.3]. Take $g \in X^{\prime}$ with $\|g\|_{X^{\prime}} \leqslant 1$. Let $B:=\|M\|_{X^{\prime} \rightarrow X^{\prime}}$ and define a function

$$
R g(x):=\sum_{k=0}^{\infty} \frac{M^{k} g(x)}{(2 B)^{k}} \quad\left(g \in X^{\prime}\right)
$$

where

$$
M^{k} g:= \begin{cases}|g| & (k=0), \\ M g & (k=1), \\ M\left(M^{k-1} g\right) & (k \geqslant 2) .\end{cases}
$$

For every $g \in X^{\prime}$ with $\|g\|_{X^{\prime}} \leqslant 1$, the function $R g$ satisfies the following properties:

1) $|g(x)| \leqslant R g(x)$ for almost every $x \in \mathbb{R}^{n}$;

2) $\|R g\|_{X^{\prime}} \leqslant 2\|g\|_{X^{\prime}} \leqslant 2$;

3) $M(R g)(x) \leqslant 2 B R g(x)$, that is, $R g$ is a Muckenhoupt $A_{1}$ weight with the $A_{1}$ constant less than or equal to $2 B$. 
By Lemma 2.3, there exist positive constants $q>1$ and $C$ independent of $g$ such that for all cubes $Q$,

$$
\left(\frac{1}{|Q|} \int_{Q} R g(x)^{q} d x\right)^{1 / q} \leqslant \frac{C}{|Q|} R g(Q) .
$$

By virtue of the generalized Hölder inequality, we obtain

$$
\begin{aligned}
\left\|(R g) \chi_{Q}\right\|_{L^{q}\left(\mathbb{R}^{n}\right)}=|Q|^{1 / q}\left(\frac{1}{|Q|} \int_{Q} R g(x)^{q} d x\right)^{1 / q} & \leqslant|Q|^{1 / q} \cdot \frac{C}{|Q|} R g(Q) \leqslant \\
& \leqslant C|Q|^{-1 /\left(q^{\prime}\right)}\|R g\|_{X^{\prime}}\left\|\chi_{Q}\right\|_{X} \leqslant C|Q|^{-1 /\left(q^{\prime}\right)}\left\|\chi_{Q}\right\|_{X} .
\end{aligned}
$$

Thus we have

$$
\begin{aligned}
\int_{Q}|f(x) g(x)| d x \leqslant \int_{Q}|f(x)| R g(x) d x \leqslant\left\|f \chi_{Q}\right\|_{L^{q^{\prime}\left(\mathbb{R}^{n}\right)}}\left\|(R g) \chi_{Q}\right\|_{L^{q}\left(\mathbb{R}^{n}\right)} & \\
& \leqslant C\left(\frac{1}{|Q|} \int_{Q}|f(x)|^{q^{\prime}} d x\right)^{1 /\left(q^{\prime}\right)}\left\|\chi_{Q}\right\|_{X} .
\end{aligned}
$$

By Lemma 2.1 we get

$$
\begin{aligned}
\left\|f \chi_{Q}\right\|_{X}=\left\|f \chi_{Q}\right\|_{X^{\prime \prime}} \leqslant C \sup \left\{\left|\int_{Q} f(x) g(x) d x\right|: g \in X^{\prime},\|g\|_{X^{\prime}} \leqslant 1\right\} \leqslant & \\
& \leqslant C\left(\frac{1}{|Q|} \int_{Q}|f(x)|^{q^{\prime}} d x\right)^{1 /\left(q^{\prime}\right)}\left\|\chi_{Q}\right\|_{X} .
\end{aligned}
$$

Consequently, the right-hand side inequality follows with $p=q^{\prime}$.

4. Examples. The authors have considered generalization of the equivalent BMO norm and proved the following statements.

1. (Izuki [20]) The variable Lebesgue norm $\|f\|_{L^{p(\cdot)\left(\mathbb{R}^{n}\right)}}$ is defined by

$$
\|f\|_{L^{p(\cdot)\left(\mathbb{R}^{n}\right)}}:=\inf \left\{\lambda>0: \int_{\mathbb{R}^{n}}\left|\frac{f(x)}{\lambda}\right|^{p(x)} d x \leqslant 1\right\} .
$$

Kováčik and Rákosník [21] have proved that the generalized Lebesgue space $L^{p(\cdot)}\left(\mathbb{R}^{n}\right)$ with variable exponent $p(\cdot)$ is a Banach function space and the associate space is $L^{p^{\prime}(\cdot)}\left(\mathbb{R}^{n}\right)$ with norm equivalence, where $p^{\prime}(\cdot)$ is the conjugate exponent given by $\frac{1}{p(\cdot)}+\frac{1}{p^{\prime}(\cdot)}=1$.

The generalized Lebesgue space $L^{p(\cdot)}\left(\mathbb{R}^{n}\right)$ consists of all measurable functions $f$ such that the norm $\|f\|_{L^{p(\cdot)\left(\mathbb{R}^{n}\right)}}$ is finite.

By using a bounded measurable function $p(\cdot): \mathbb{R}^{n} \rightarrow[1, \infty)$ we generalize the semi-norm (1.3) to

$$
\|b\|_{\mathrm{BMO}_{L^{p(\cdot)}}}:=\sup _{Q: \text { cube }} \frac{1}{\left\|\chi_{Q}\right\|_{L^{p(\cdot)}\left(\mathbb{R}^{n}\right)}}\left\|\left(b-b_{Q}\right) \chi_{Q}\right\|_{L^{p(\cdot)}\left(\mathbb{R}^{n}\right)} .
$$


If $p(\cdot)$ satisfies $p_{-}>1$ and the Hardy-Littlewood maximal operator $M$ is bounded on $L^{p(\cdot)}\left(\mathbb{R}^{n}\right)$, then the generalized BMO norm $\|b\|_{\mathrm{BMO}_{L^{p(\cdot)}}}$ is equivalent to the classical one $\|b\|_{\text {BMO }}$.

2. (Izuki and Sawano [22]) If a bounded measurable function $p(\cdot): \mathbb{R}^{n} \rightarrow[1, \infty)$ satisfies $1 \leqslant \inf p(x)$ and the log-Hölder conditions:

$$
\begin{gathered}
|p(x)-p(y)| \leqslant \frac{C}{-\log (|x-y|)} \quad \text { for } \quad x, y \in \mathbb{R}^{n},|x-y| \leqslant 1 / 2, \\
\left|p(x)-p_{\infty}\right| \leqslant \frac{C}{\log (e+|x|)} \quad \text { for } \quad x \in \mathbb{R}^{n},
\end{gathered}
$$

for some constants $C$ and $p_{\infty}$ independent of $x, y$, then $\|b\|_{\mathrm{BMO}_{L^{p(\cdot)}}}$ is equivalent to $\|b\|_{\text {ВMO. }}$

3. (Izuki, Sawano and Tsutsui [14]) If a variable exponent $p(\cdot): \mathbb{R}^{n} \rightarrow[1, \infty)$ is bounded and $M$ is of weak type $(p(\cdot), p(\cdot))$, that is, there exists a constant $C>0$ such that for all $f \in L^{p(\cdot)}\left(\mathbb{R}^{n}\right)$ and all $\lambda>0$,

$$
\left\|\chi_{\{M f>\lambda\}}\right\|_{L^{p(\cdot)}\left(\mathbb{R}^{n}\right)} \leqslant C \lambda^{-1}\|f\|_{L^{p(\cdot)}\left(\mathbb{R}^{n}\right)},
$$

then $\|b\|_{\mathrm{BMO}_{L}(\cdot)}$ is equivalent to $\|b\|_{\mathrm{BMO}}$.

4. (Ho [4]) Ho obtained a characterization in the context of general function space including Lebesgue spaces. Given a ball Banach function space $X$ equipped with a norm $\|\cdot\|_{X}$, we define the $X$-based generalized BMO norm

$$
\|b\|_{\mathrm{BMO}_{X}}:=\sup _{Q: \text { cube }} \frac{1}{\left\|\chi_{Q}\right\|_{X}}\left\|\left(b-b_{Q}\right) \chi_{Q}\right\|_{X} .
$$

If $M$ is bounded on the associate space $X^{\prime}$, then $\|b\|_{\mathrm{BMO}_{X}}$ is equivalent to $\|b\|_{\mathrm{BMO}}$. We remark that Ho's results [4, 23] have included the authors' one [22, 24]. The statements in $[22,24]$ are deeply depending on Diening's work [25] on variable exponent analysis. On the other hand, Ho's proof is self-contained and obtained as a by-product of the new results about atomic decomposition introduced in [4]. Our proof of the result, initially proved by Ho, is new in the sense that we use the Rubio de Francia algorithm [26-28].

The authors are thankful to the anonymous referee for his/her careful reading of this paper. The authors are appreicate to Professor Eiichi Nakai for his introducing the papers [5-8]. Finally, the authors thank Professor Tuomas Hytönen for his information on the paper [17] as well as (2.3).

Received: March 28, 2016; accepted: October 6, 2016.

Authors information

Mitsuo Izuki - Senior Assistant Professor; izuki@okayama-u.ac.jp

Yoshihiro Sawano - Associate Professor; ysawano@tmu.ac.jp 


\section{References}

1. John J., Nirenberg L., "On functions of bounded mean oscillation", Comm. Pure Appl. Math. 14, 415-426 (1961).

2. Hakim D.-I., Sawano Y., "Interpolation of generalized Morrey spaces", Rev. Mat. Complut. 29, 295-340 (2016).

3. Bennett C., Sharpley R., Interpolations of Operators (Academic Press, New York, 1988).

4. Ho K.-P., "Atomic decomposition of Hardy spaces and characterization of BMO via Banach function spaces", Anal. Math. 38, 173-185 (2012).

5. Campanato S., "Proprietà di hölderianità di alcune classi di funzioni", Ann. Scuola Norm. Sup. Pisa 17(3) 175-188 (1963) [In Italian].

6. Meyers N. G., "Mean oscillation over cubes and Hölder continuity", Proc. Amer. Math. Soc. 15, 717-721 (1964).

7. Peetre J., "On the theory of Lp, $\lambda$ spaces", J. Funct. Anal. 4, 71-87 (1969). (2008).

8. Nakai E., "A generalization of Hardy spaces Hp by using atoms", Acta Math. Sin. 24, 1243-1268

9. Okada S., Ricker W., Sánchez Pérez E., Optimal Domain and Integral Extension of Operators (Birkhäuser, Basel, 2008).

10. Sawano Y., Tanaka H., "The Fatou property of block spaces", J. Math. Sci. Univ. Tokyo 22, $663-683$ (2015).

11. Zorko C. T., "Morrey space", Proc. Amer. Math. Soc. 98, 586-592 (1986).

12. Sawano Y., Tanaka H., "Predual spaces of Morrey spaces with non-doubling measures", Tokyo J. Math. 32, 471-486 (2009).

13. Izuki M., "Remarks on Muckenhoupt weights with variable exponent", J. Anal. Appl. 11, 27-42 (2013).

14. Izuki M., Sawano Y., Tsutsui Y., "Variable Lebesgue norm estimates for BMO functtions. II", Anal. Math. 40, 215-230 (2014).

15. Duoandikoetxea J., Fourier Analysis In Ser. Graduate Studies in Math. (Amer. Math. Soc., Providence, RI, 29, 2001).

16. Grafakos L., Modern Fourier analysis. Second edition In Ser. Graduate Texts in Mathematics (Springer, New York, 250, 2009).

17. Hytönen T., Pérez C., "Sharp weighted bounds involving $A_{\infty}$ ", Anal. PDE 6, 777-818 (2013).

18. Pérez C., Rela E., "A new quantitative two weight theorem for the Hardy-Littlewood maximal operator", Proc. Amer. Math. Soc. 143(2), 641-655 (2015).

19. Cruz-Uribe D., Hernández E., Martell J. M., "Greedy bases in variable Lebesgue spaces", Monatsh. Math. 179, 355-378 (2016).

20. Izuki M., "Boundedness of commutators on Herz spaces with variable exponent", Rend. Circ. Mat. Palermo 59(2), 199-213 (2010).

21. Kováčik O., Rákosník J., "On spaces $L p(x)$ and $W k, p(x)$ ", Czech. Math. J. 41, $592-618$ (1991).

22. Izuki M., Sawano Y., "Variable Lebesgue norm estimates for BMO functions", Czechoslovak Math. J. 62, 717-727 (2012).

23. Ho K.-P., "Vector-valued John-Nirenberg inequalities and vector-valued mean oscillations characterization of BMO", Results Math. 70(1-2), 257-270 (2016).

24. Diening L., "Maximal functions on Musielak-Orlicz spaces and generalized Lebesgue spaces", Bull. Sci. Math. 129, 657-700 (2005).

25. Rubio de Francia J. L., "Factorization and extrapolation of weights", Bull. Amer. Math. Soc. 7, 393-395 (1982).

26. Rubio de Francia J. L., "A new technique in the theory of Ap weights", Proc. of a seminar held in Turin and Milan, 1982 "Topics in modern harmonic analysis", 571-579 (Ist. Naz. Alta Mat. Francesco Severi, Rome, 1983). (1984).

27. Rubio de Francia J. L., "Factorization theory and Ap weights", Amer. J. Math. 106, 533-547

28. Garling D.J.H., Inequalities: a journey into linear analysis (Cambridge University Press, Cambridge, 2007).

For citation: Mitsuo Izuki, Yoshihiro Sawano. Characterization of BMO via ball Banach function spaces. Vestnik SPbSU. Mathematics. Mechanics. Astronomy, 2017, vol. 4(62), issue 1, pp. 78-86. DOI: $10.21638 / 11701 / \mathrm{spbu01.2017.110}$ 